\title{
CALCULATION OF PHASE DIAGRAMS AND THE EFFECT OF LATTICE PARAMETERS ON THE HIGH-ENTROPY CARBIDES SINGLE-PHASE SOLID SOLUTIONS FORMATION
}

\author{
Tagir MAKHMUTOV, Nikolay RAZUMOV, Anatoliy POPOVICH \\ Peter the Great St. Petersburg Polytechnic University, St. Petersburg, Russian Federation, \\ mahmutovtagir@mail.ru
}

https://doi.org/10.37904/metal.2021.4270

\begin{abstract}
This paper presents the results of phase composition calculating by the CALPHAD method using the ThermoCalc software package for thermodynamic calculations of high-entropy carbides (TiZrHfTaNb)C, (TiZrHfTaNbW)C, (TiZrHfTaNbMo)C. The starting materials lattice parameters influence the possibility of highentropy carbides single-phase solid solutions formation. A comparative analysis of lattice parameters influence and phase diagrams was carried out. An increase in the difference between the lattice parameters decreases the homogeneity region of carbide solid solutions with the crystal structure of rock salt $(\mathrm{NaCl})$.
\end{abstract}

Keywords: High entropy alloys, carbides, Thermo-Calc, phase diagrams

\section{INTRODUCTION}

Ceramics based on borides and carbides of high-entropy alloys (HEA) are the new class of materials that are of the highest interest of the world scientific community. Multicomponent ceramics demonstrate higher hardness, wear-resistance, and oxidation resistance than carbides and borides of pure metals $[1,2]$. The development and wide research interest in high-entropy ceramics among scientists initially awakened "highentropy alloys," the theory of which states that the entropy of mixing in multicomponent systems stabilizes solid solutions, preventing the formation of intermetallic phases [3]. Later studies of HEA showed that the entropy of mixing is not the only factor influencing the formation of a solid solution. Therefore, methods have been proposed for assessing the stabilization of solid solutions, taking into account the effect of atomic radii [4], the concentration of valence electrons [5], the general effect of entropy, enthalpy of mixing, and average melting point [6]. However, these methods cannot be applied to carbides, nitrides, and borides of high-entropy alloys. The lattice parameter of the corresponding compounds is used instead of the atomic radii of the elements to describe the distortion of the crystal structure of high-entropy ceramics. The enthalpy of mixing is determined under the condition that the multicomponent system consists of individual compounds [7-9].

Under actual conditions, diborides are formed in an anisotropic hexagonal structure of the $\mathrm{AlB}_{2}$ type. Each metal atom gives up two electrons to form a bond with boron Me-B, which has mixed covalent and ionic characteristics. The Me-Me bonds are deformed by boron atoms inside the layers, forming a rigid network [10].

High-entropy carbides in most of the studies carried out, are represented by metals of subgroup 4 (Ti, $\mathrm{Zr}, \mathrm{Hf}$ ) and subgroup $5(\mathrm{~V}, \mathrm{Nb}, \mathrm{Ta})$ of the periodic table. Metals that form monocarbides with a cubic structure such as $\mathrm{NaCl}$, where metals are most likely have a common cationic sublattice with the face-centred cubic (fcc) sublattice, and carbon $C$ is located in the anionic fcc sublattice [11]. Our previous work evaluated the effect of surface carbon saturation of the CrNbMoWV alloy on hardness, corrosion, and tribological properties [12].

According to the rule of atomic size influence, if the difference between the atomic radius of soluble and a solvent in a binary system does not exceed $15 \%$, complete dissolution and the formation of a solid solution are possible. This rule was extrapolated to multicomponent systems, defined and calculated as the difference 
in atomic sizes - $\delta$, as shown in Equation $\mathbf{1}$ (where $n$ is the total number of elements, and $c_{i}$ and $r_{i}$ are the composition and atomic size of the $\mathrm{i}^{\text {th }}$ element, respectively) [4].

$\delta=\sqrt{\sum_{i=1}^{n} c_{i}\left(1-\frac{r_{i}}{r_{\mathrm{cp}}}\right)^{2}}$

The mean deviation of atomic radii is an important empirical parameter used to predict the formation of singlephase solid solutions in high-entropy alloys [13]. However, this method cannot be applied to carbides, nitrides, and borides of high-entropy alloys. The lattice parameter values of the corresponding carbides are used to describe the distortion of the crystal structure in carbides instead of the elements atomic radii. Since metal atoms migrate only to the nearest metal vacancy on their initial planes, it is assumed that the migration of metal atoms occurs only in close-packed planes. Since $\mathrm{HfC}, \mathrm{TaC}$ and TiC have a crystal structure of rock salt $(\mathrm{NaCl})$, which contains 8 atoms per cell, $a^{\prime}$ was calculated based on the reduced primitive cell by Equation 2 , while $a^{\prime}$ for $\mathrm{Mo}_{2} \mathrm{C}$ and $\mathrm{WC}$ remains the same as its lattice parameter $a$ [9].

$a^{\prime}=\sqrt{2} a / 2$

The average difference between the lattice parameters is determined by the following equation:

$\delta_{a^{\prime}}=\sqrt{\sum_{i=1}^{N} X_{i}\left[1-a_{i}^{\prime} /\left(\sum_{i=1}^{N} X_{i} a_{i}^{\prime}\right)\right]^{2}}$

where $a_{i}^{\prime}$ is the lattice parameters of the $i^{\text {th }}$ component carbide, $X_{i}$ is the components molar fraction. The value of the parameter $\delta_{a^{\prime}}$ must be less than $6.6 \%$ for the formation of a solid solution [8].

\section{MATERIALS AND METHODS}

Three systems were chosen for the calculations: (TiZrHfTaNb)C, (TiZrHfTaNbW)C, (TiZrHfTaNbMo)C. The phase diagrams were calculated by the CALPHAD method using the Thermo-Calc software package for thermodynamic calculations based on the HEA4 high-entropy alloys database in console mode. A temperature range of $500-4000 \mathrm{~K}$ was chosen for the calculation, the carbon content varied from 0 to 1 molar fractions, and the balance for all elements.

Table 1 Lattice parameter of carbides

\begin{tabular}{|c|c|c|}
\hline Component & Lattice parameters $\boldsymbol{a}(\mathrm{nm})[14]$ & Lattice length at the closed-packed plane $\boldsymbol{a}^{\prime}$ \\
\hline $\mathrm{ZrC}$ & 4.73 & 3.34 \\
\hline $\mathrm{HfC}$ & 4.65 & 3.29 \\
\hline $\mathrm{TiC}$ & 4.35 & 3.08 \\
\hline $\mathrm{TaC}$ & 4.45 & 3.15 \\
\hline $\mathrm{NbC}$ & 4.47 & 3.16 \\
\hline $\mathrm{WC}$ & 2.90 & 2.90 \\
\hline $\mathrm{Mo}_{2} \mathrm{C}$ & 2.99 & 2.99 \\
\hline
\end{tabular}

The calculation of $a^{\prime}$ was carried out according to the Equation (2), the calculation of the average difference was carried out according to the Equation (3). The cell parameters for all carbides are given in Table 1.

\section{RESULTS AND DISCUSSION}

The phase diagram of the (TiZrHfTaNb)C system high-entropy carbide is shown in Figure 1. According to the diagram obtained, in the range of carbon contents up to 0.35 molar fractions, the formation of three main 
phases are possible, two high-entropy bcc solid solutions of various compositions. The third phase corresponds to a solid solution with carbon and is designated DIS_FCC_A1\#2 (crystal structure of rock salt $(\mathrm{NaCl}))$. The content of DIS_FCC_A1\#2 increases with increasing carbon content and reaches its maximum value at 0.5 mole fractions of carbon. A pure high-entropy solid solution of carbides with a $\mathrm{NaCl}$-type structure has a wide range of homogeneity in temperature and carbon content. The results agree with the calculation of the lattice parameters influence $\delta_{a}=3.06 \%$ (Table 2), which also confirms the possibility of carbides solid solution formation.

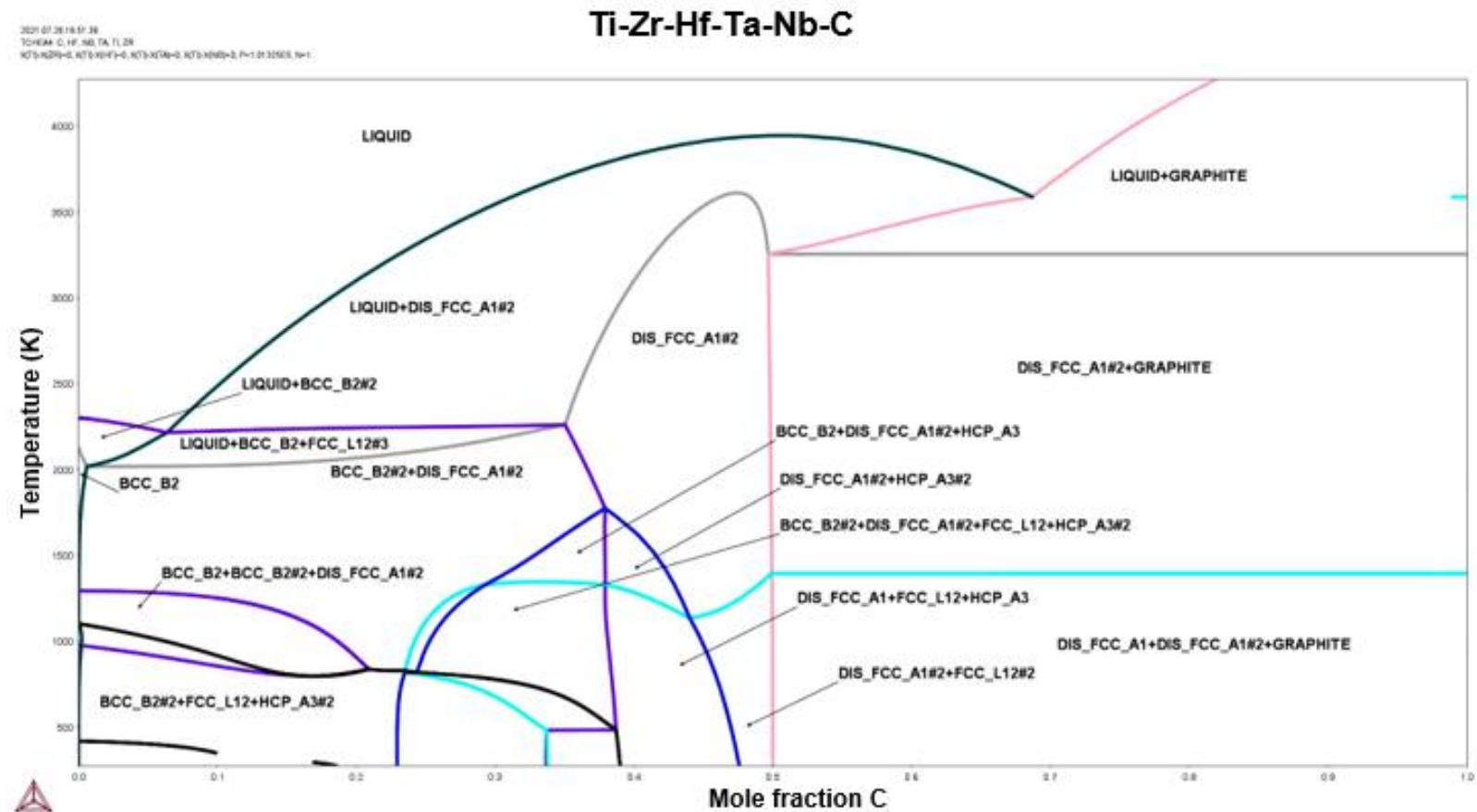

Figure 1 Phase diagram of the (TiZrHfTaNb)C system

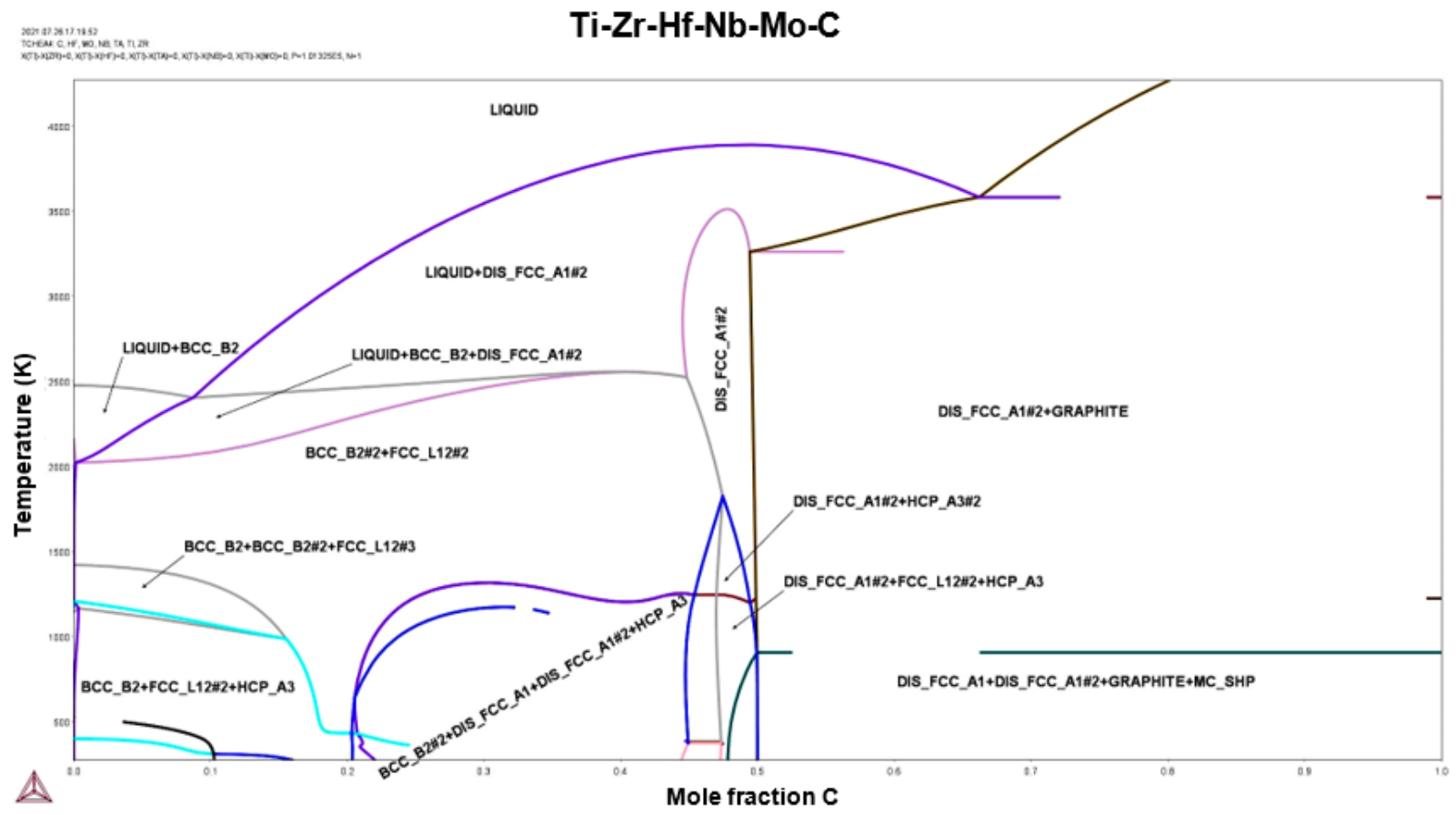

Figure 2 Phase diagram of the (TiZrHfTaNbMo)C system 
The phase diagram of the (TiZrHfTaNbMo)C system high-entropy carbide is shown in Figure 2. According to the diagram, the addition of molybdenum narrows the homogeneity region of the DIS_FCC_A1\#2 solid solution and is in equilibrium with the maximum carbon content in the range of $0.45-0.5$ mole fractions. Thus, molybdenum shifts the equilibrium towards the formation of solid solutions with a bcc lattice. The addition of molybdenum also increases the lattice influence parameter $\delta_{a^{\prime}}=3.75 \%$ (Table 2), but does not exclude the possibility of the high-entropy carbides solid solution formation with the $\mathrm{NaCl}$ type structure.

The phase diagram of the (TiZrHfTaNbW) C system high-entropy carbide is shown in Figure 3. According to the diagram, the addition of tungsten narrows the homogeneity region of the DIS_FCC_A1 \# 2 solid solution in comparison with the (TiZrHfTaNb) $\mathrm{C}$ and (TiZrHfTaNbMo) $\mathrm{C}$ system, especially at temperatures below 3000 $\mathrm{K}$. The influence of the lattice size also increases and is $\delta_{a}=4.58 \%$ (Table 2), but does not exclude the possibility of the high-entropy carbides solid solution formation with the $\mathrm{NaCl}$ type structure.

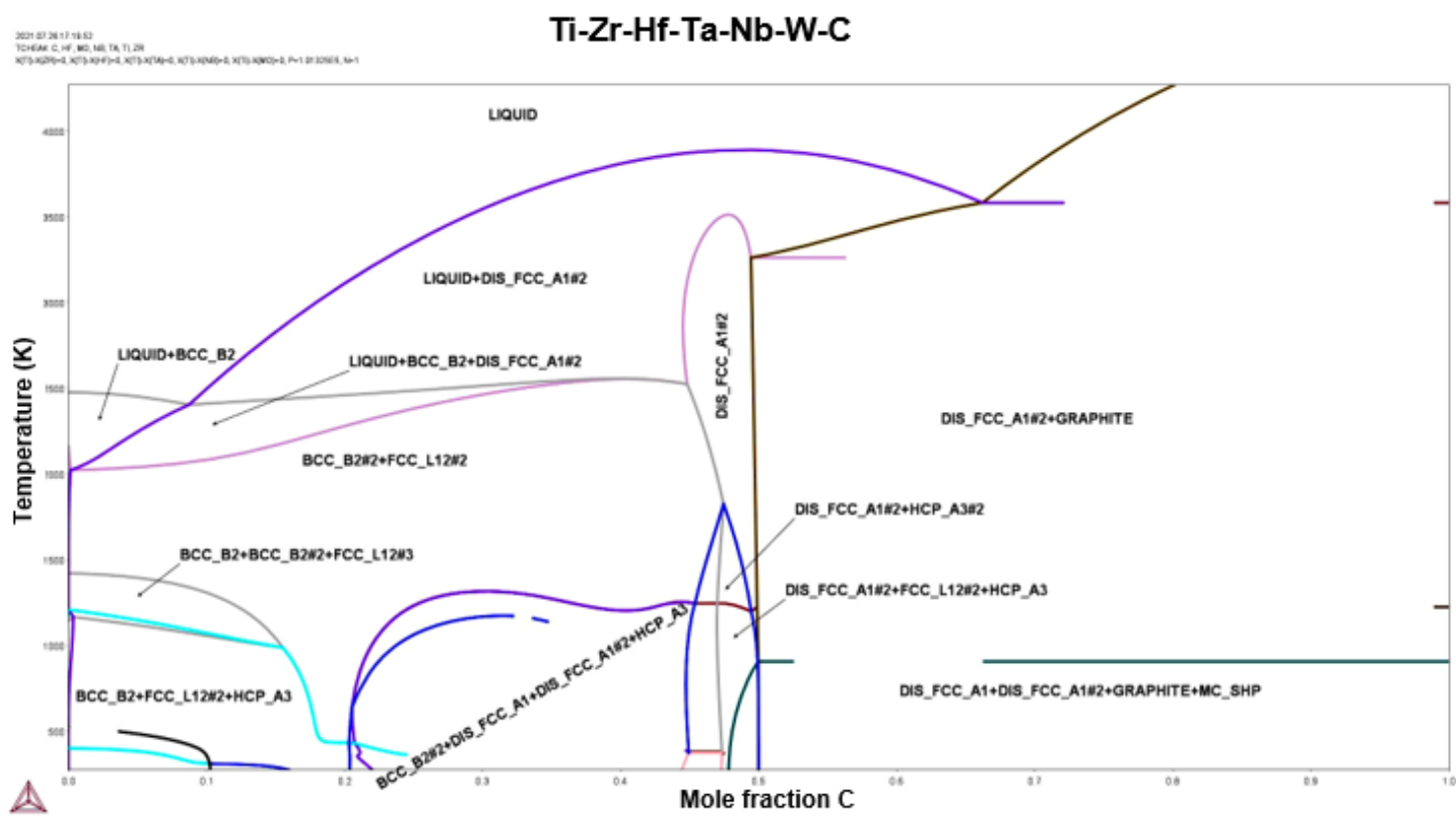

Figure 3 Phase diagram of the (TiZrHfTaNbW)C system

The obtained phase diagrams of high-entropy carbides solid solutions agree with the calculated values of the lattice parameters influence on the formation of solid solutions and do not exceed the critical value of the component $\delta_{a^{\prime}}=6.6 \%$.

Table 2 Lattice parameters of high-entropy carbides (HEC)

\begin{tabular}{|c|c|c|}
\hline HEC & $\boldsymbol{X}_{i}$, mole fraction & $\boldsymbol{\delta}_{\mathbf{a}^{\prime} \%}$ \\
\hline$($ TiZrHfTaNb)C & 0.2 & 3.06 \\
\hline$($ TiZrHfTaNbMo)C & 0.1667 & 3.75 \\
\hline (TiZrHfTaNbW)C & 0.1667 & 4.58 \\
\hline
\end{tabular}

\section{CONCLUSION}

In the presented work, the high-entropy carbides phase diagrams of the (TiZrHfTaNb)C, (TiZrHfTaNbW)C, and (TiZrHfTaNbMo)C systems were calculated. In addition, calculations of the influence of initial carbides lattice parameters on the high-entropy carbides solid solution formation have been performed. It was found that the two prediction methods presented in the paper are consistent with each other. The largest homogeneity region 
is characteristic for the (TiZrHfTaNb)C system; the difference in the lattice parameters is $\delta_{a^{\prime}}=3.06 \%$. With the addition of Mo, the lattice parameters difference increases to $3.75 \%$, and with the addition of $\mathrm{W}$, it increases to $4.58 \%$. Thus, all of the high-entropy carbides presented tend to form single-phase solid solutions.

\section{ACKNOWLEDGEMENTS}

\section{The research was supported by the Ministry of Science and Higher Education of the Russian Federation (State Assignment for basic research 0784-2020-0022).}

\section{REFERENCES}

[1] KAN, W. H., et al. Precipitation of $(\mathrm{Ti}, \mathrm{Zr}, \mathrm{Nb}, \mathrm{Ta}, \mathrm{Hf}) \mathrm{C}$ high entropy carbides in a steel matrix. Materialia. 2020, vol. 9, no. 100540.

[2] BRAIC, V., VLADESCU, A., BALACEANU, M., LUCULESCU, C. R., BRAIC, M. Nanostructured multi-element (TiZrNbHfTa)N and (TiZrNbHfTa)C hard coatings. Surf. Coatings Technol. 2012, vol. 211, pp. 117-121.

[3] MURTY, B.S., YEH, J.W., RANGANATHAN, S., BHATTACHARJEE, P.P. High-Entropy Alloys. Elsevier, 2019.

[4] ZHANG, Y., ZHOU, Y.J., LIN, J.P., CHEN, G.L., LIAW, P.K. Solid-solution phase formation rules for multicomponent alloys. Adv. Eng. Mater. 2008, vol. 10, no. 6, pp. 534-538.

[5] GUO, S., NG, C., LU J., LIU, C.T. Effect of valence electron concentration on stability of fcc or bcc phase in high entropy alloys. J. Appl. Phys. 2011, vol. 109, no. 10, p. 103505.

[6] ZHANG, Y., YANG, X., LIAW, P.K. Alloy design and properties optimization of high-entropy alloys. JOM. 2012, vol. 64 , no. 7 , pp. 830-838.

[7] WANG, K., et al. Microstructure and mechanical properties of (TiZrNbTaMo)C high-entropy ceramic. J. Mater. Sci. Technol. 2020, vol. 39, pp. 99-105.

[8] YANG, Y., WANG, W., GAN, G.Y., SHI, X.F., TANG, B.Y. Structural, mechanical and electronic properties of (TaNbHfTiZr)C high entropy carbide under pressure: Ab initio investigation. Phys. B Condens. Matter. 2018, vol. 550, no. August, pp. 163-170.

[9] ZHANG, H., HEDMAN, D., FENG, P., HAN, G., AKHTAR, F. A high-entropy B4(HfMo2TaTi)C and SiC ceramic composite. Dalt. Trans. 2019, vol. 48, no. 16, pp. 5161-5167.

[10] GILD, J., et al. High-entropy metal diborides: A new class of high-entropy materials and a new type of ultrahigh temperature ceramics. Sci. Rep. 2016, vol. 6, pp. 2-11.

[11] WANG, F., et al. Irradiation damage in $(\mathrm{Zr0} .25 \mathrm{Ta} 0.25 \mathrm{Nb} 0.25 \mathrm{Ti} 0.25) \mathrm{C}$ high-entropy carbide ceramics. Acta Mater. 2020, vol. 195, pp. 739-749.

[12] RAZUMOV, N., et al. Refractory CrMoNbWV high-entropy alloy manufactured by mechanical alloying and spark plasma sintering: Evolution of microstructure and properties. Materials (Basel). 2021, vol. 14, no. 3, pp. 1-14.

[13] SRIHARITHA, R., MURTY, B.S., KOTTADA, R.S. Phase formation in mechanically alloyed Al $x$ CoCrCuFeNi $(x=$ $0.45,1,2.5,5$ mol) high entropy alloys. Intermetallics. 2013, vol. 32, pp. 119-126.

[14] http://www.crystallography.net/cod/index.php. 